\title{
Willingness to Accept a COVID-19 Vaccine in Nigeria: A Population-based Cross-sectional Study
}

\author{
Ekaete Alice Tobin $^{1,2, *}$, Martha Okonofua ${ }^{1}$, Azuka Adeke $^{3}$, Andrew Obi ${ }^{4,5}$ \\ ${ }^{1}$ Institute of Lassa Fever Research and Control, Irrua Specialist Teaching Hospital, Irrua, Nigeria \\ ${ }^{2}$ Ogwa Comprehensive Health Centre, Ogwa, Nigeria \\ ${ }^{3}$ Department of Community Medicine, Alex Ekwueme Federal University Teaching Hospital, Abakaliki, Nigeria \\ ${ }^{4}$ Department of Community Health, University of Benin, Benin-city, Nigeria \\ ${ }^{5}$ Department of Community Health, University of Benin Teaching Hospital, Benin-City, Nigeria
}

Email address:

ekaete.tobin@gmail.com (E. A. Tobin), Okonofuamartha9@gmail.com (M. Okonofua), Azukaadeke@gmail.com (A. Adeke),

Andrew.obi@uniben.edu (A. Obi)

${ }^{*}$ Corresponding author

\section{To cite this article:}

Ekaete Alice Tobin, Martha Okonofua, Azuka Adeke, Andrew Obi. Willingness to Accept a COVID-19 Vaccine in Nigeria: A Population-based Cross-sectional Study. Central African Journal of Public Health. Vol. 7, No. 2, 2021, pp. 53-60.

doi: $10.11648 /$ j.cajph.20210702.12

Received: February 22, 2021; Accepted: March 11, 2021; Published: March 26, 2021

\begin{abstract}
Background: The study assessed the intention to accept a future COVID-19 vaccine in Nigeria and associated factors. Materials and Methods: Between July 2020 and August 2020, a cross-sectional study was conducted using an online questionnaire that captured demographic data, risk perception, trust in government and public health authorities and willingness to accept a future COVID-19 vaccine. Data were analyzed using Statistical Package for Social Science version 21.0, Chi-square and logistic regression were carried out at a 95\% confidence interval. Appropriate institutional ethical board approval and informed consent were obtained from all participants. Results: 1,228 responses were received over the study period. The mean age of respondents was 32.8 years (SD 10.4), 12.7\% $(156 / 1,228)$ were health workers, $70.1 \%(861 / 1,228)$ had tertiary level of education. Intention to accept a future COVID-19 vaccine was expressed by $50.2 \%(617 / 1,228)$ of respondents. Increasing age, male gender, trust in government, trust in public health authorities, confidence in vaccine developers, willingness to pay for and travel for a vaccine, and vaccination during an outbreak were significantly associated with COVID-19 vaccine acceptance. Healthcare workers and respondents with pre-existing medical conditions were not significantly different from non-healthcare workers and persons without medical conditions respectively with regards to the willingness to be vaccinated. Conclusion: One in two persons would accept a COVID-19 vaccine when it becomes available in the country. The government should take pro-active steps to address the factors that may potentially impact on the benefits expected from the introduction of COVID-19 vaccine and scale-up vaccine sensitization to improve potential acceptance for uptake across the country.
\end{abstract}

Keywords: COVID-19, Hesitancy, Intention, Vaccine, Willingness

\section{Introduction}

The novel coronavirus disease (COVID-19) caused by the SARS-Coronavirus-2 (SARS-CoV-2) was declared a global pandemic on $11^{\text {th }}$ March 2020 by the World Health Organization and has swept across all continents of the world, affecting over 213 countries and territories [1]. The pandemic poses a serious threat to global public health, socio-economic stability, food security, trade and industry with the impact felt in high and low-income countries alike. [2] Since the first case was detected in Egypt on the $14^{\text {th }}$ day of February 2020, the number of cases in Africa has been on a steady rise, though has remained lower than the rest of the world. [3] With over 1.3 billion people and a weak health system plagued by lack of healthcare infrastructure and shortages of health manpower, limited access to social protection and low health literacy, the public health measures implemented at the start of the pandemic will not be sufficient to stop further progress of the 
virus in Africa or end the pandemic. A COVID-19 vaccine may be the most practical and feasible solution for Africa. Several vaccine candidates are currently under different stages of development and some may be available for phase 3 trials before the end of 2020. [4]

While the focus is more on the development of a COVD-19 vaccine, less attention has been paid to the extent to which a vaccine will be accepted in Africa. The introduction of a new health intervention is not necessarily followed by acceptance and adoption by communities. There are several, demographic, individual, socio-political, financial, and cultural dimensions that interplay to influence the adoption and implementation of new health interventions.

Few studies conducted on acceptance of a COVID-19 vaccine have produced varied results with rates as low as $37 \%$ and as high as $86 \%$. [5]

Nigeria is a multi-ethnic, multi-cultural and multi-religious country. Experiences with the GAVI-supported routine immunization program shows vaccination coverage rates differ across the country with higher coverage rates in the southern states compared to the north, and within states, higher coverage rates in urban compared to rural areas. Disparities in rates are also observed when comparison is made across caretaker literacy levels, family wealth index and caretaker age. [6] The polio vaccination refusal saga in 3 states in northern Nigeria between 2003 and 2004 is a grim reminder of how public mistrust of government intentions and the international community, political and religious discordances and poor community engagement can disrupt a vaccination program intended for the good of the people with grave consequences. [7] It stresses the need for a clear understanding of the context-specific factors that may influence a COVID-19 vaccination program in Nigeria, and the timely implementation of strategies to achieve high coverage rates when a vaccine eventually becomes available in the country. The study objectives were to investigate the intention to accept a future COVID-19 vaccine in Nigeria and to determine the factors associated with intent to accept or refuse the vaccine.

\section{Methodology}

\subsection{Study Area and Population}

The study was carried out in Nigeria, located on the West coast of Africa with boundaries as Niger, Chad, Cameroon, Benin and the Gulf of Guinea. The country covers a landmass of 923,768 square kilometres and is divided into 6 geopolitical zones: North-west, North-central, North-east, South-south, South-east and South-west. With a projected population of $200,000,000$ and the annual population growth rate of $2.38 \%$, the country is the most populous in West Africa.

The study population were Nigerians with eligibility as access to the internet, willingness to consent, age above 18 years of age and current place of residence as Nigeria.

The survey utilized a cross-sectional study design with a minimum sample size of 1,067 calculated based on the assumption of a $50 \%$ vaccine acceptance rate, a $3 \%$ margin of error and a confidence interval of $95 \%$. The survey was conducted between June and August 2020.

\subsection{Sampling}

The restrictions on movement and recommendation to maintain physical distancing that were in force at the time the study was conducted did not permit face-to-face recruitment of study participants. Instead, a simplified snowballing technique was used where the link to an online survey tool was distributed on the WhatsApp social media platform to all contacts of the researchers. [8] Recipients were encouraged to forward the invitation and link to their WhatsApp contacts and contacts on other social media networks.

\subsection{Data Collection}

Data were collected using a structured English language questionnaire created on google forms and shared through a link on WhatsApp. The questionnaire was designed from a literature search and inclusion of questions from the SAGE vaccine hesitancy survey. [9] The introductory part of the questionnaire contained informed consent with a brief introduction to the study and study objectives.

The questionnaire was structured in five thematic sections. The first section collected socio-demographic information including age, sex, marital status, religion, educational level, employment status and occupation and any pre-existing illness specifically hypertension, diabetes mellitus, cancer, human immunodeficiency disease, asthma, kidney disease or heart disease. Occupation was categorized as healthcare provider and non-healthcare provider.

The second section had 3 questions on basic vaccine knowledge, capturing the purpose of vaccination, mode of action and relevance in outbreaks, with responses as 'Yes', 'No' and 'I don't know'. Responses were coded as Yes $=1$, No or I don't know $=0$. Level of basic knowledge was dichotomized as good or inadequate based on a score of 3-4 or 0-2 respectively.

Section three assessed the socio-political dimensions of vaccine acceptance with 6 questions on who the vaccine should be made compulsory for, trust in vaccine manufacturers, government, and public health authorities and risk perception. To assess trust, respondents were instructed to select one of 3 responses: 'Yes', 'No' and 'I don't know'. Perceived risk was assessed on a Likert scale as: 'very high risk', 'high risk', 'low risk', and 'no risk'. All Likert-scale responses were dichotomized as 'strongly agree/ agree' $=1$ and 'Uncertain/disagree/Strongly disagree' $=0$ [10]. Perception of risk was dichotomized as 'No risk/ low risk' $=0$ and 'High risk/very high risk' $=1$.

Section four addressed willingness to receive a hypothetical COVID-19 vaccine by the question: "Would you be willing to accept a COVID-19 vaccine when one becomes available in the country?" Respondents were asked to indicate if they would be willing to pay for a COVID-19 vaccine and willing to travel for more than one hour to get a COVID-19 vaccine, for which they had to choose from: 'Yes', 'No' and 'I am not 
sure' responses.

Section five contained 2 questions that assessed history of vaccine hesitancy.

Face and content validation of the study questionnaire was carried out to ascertain the validity of the data collection tool. The reliability of the tool was assessed using the test-retest method with the tool administered twice within a space of 2 weeks to 2 groups of 5 community residents. The Pearson moment-correlation coefficient analysis gave a reliability coefficient of 0.77 .

The exposure variables in the study included sociodemographic characteristics, trust in the public health authorities, trust in government, trust in vaccine developers, risk perception, willingness to travel to receive a vaccine, willingness to pay for a vaccine, previous vaccine hesitancy. The outcome variable was the intention to accept a future COVID-19 vaccine.

\subsection{Data Analysis}

Data analysis was carried out using Statistical Package for the Social Sciences (SPSS) version 21.0 (IBM Corporation, Armonk, NY, USA). Descriptive statistics including frequency tables, means and standard deviation were used to present categorical variables. Dichotomized responses were presented as proportions. Bivariate analysis was used to examine the association between exposure and outcome variables, and regression analysis to derive the odds ratios (OR) and their 95\% confidence intervals $(95 \% \mathrm{CI})$ for variables significant at bivariate analysis. A two-tailed p-value $<0.05$ was statistically significant.

\subsection{Ethical Considerations}

Ethical approval was received from the Irrua Specialist Teaching Hospital Ethics Committee. Information on the study was provided as the first section of the survey and potential respondents were required to click on a button to show they gave consent before proceeding to complete the questionnaire. Confidentiality was maintained as names were not required, and data was accessible only to the researchers.

Table 1. Socio-demographic characteristics of respondents $(n=1,228)$.

\begin{tabular}{|c|c|}
\hline Variable & Frequency (\%) \\
\hline \multicolumn{2}{|l|}{ Age } \\
\hline$\leq 24$ & $276(22.5)$ \\
\hline $25-34$ & $472(38.4)$ \\
\hline $35-44$ & $330(26.9)$ \\
\hline$\geq 45$ & $150(12.2)$ \\
\hline \multicolumn{2}{|l|}{ Sex } \\
\hline Male & $635(51.7)$ \\
\hline Female & $593(48.3)$ \\
\hline \multicolumn{2}{|l|}{ Religion } \\
\hline Christianity & $1013(82.5)$ \\
\hline Islam & $207(16.9)$ \\
\hline Others* & $8(0.7)$ \\
\hline \multicolumn{2}{|l|}{ Marital Status } \\
\hline Single & $589(48.0)$ \\
\hline Married & $593(48.3)$ \\
\hline Divorced/widowed/separated & $46(3.1)$ \\
\hline \multicolumn{2}{|l|}{ Educational level } \\
\hline Primary & $46(3.7)$ \\
\hline Secondary & $321(26.1)$ \\
\hline Tertiary & $861(70.1)$ \\
\hline \multicolumn{2}{|l|}{ Profession } \\
\hline Non-healthcare provider & $1072(87.3)$ \\
\hline Healthcare provider & $156(12.7)$ \\
\hline \multicolumn{2}{|l|}{ Employment status } \\
\hline Employed with government & $288(23.5)$ \\
\hline Employed in private sector & $174(14.2)$ \\
\hline Self-employed & $458(37.3)$ \\
\hline Unemployed & $308(25.1)$ \\
\hline \multicolumn{2}{|l|}{ Geopolitical zone } \\
\hline South-south & $698(56.8)$ \\
\hline South-East & $95(7.7)$ \\
\hline South-West & $92(7.5)$ \\
\hline North-Central & $219(17.8)$ \\
\hline North-West & $102(8.3)$ \\
\hline North-East & $22(1.8)$ \\
\hline \multicolumn{2}{|c|}{ Existing chronic medical condition } \\
\hline No & $1144(93.2)$ \\
\hline Yes & $84(6.8)$ \\
\hline
\end{tabular}




\section{Results}

One thousand two hundred and twenty-eight responses were received over the survey period. Mean age of respondents was 32.8 years (SD 10.4). The highest proportion was in the 25-34 years age group, 472 (38.4\%), 592 (48.3\%) were married and the majority, $861(70.1 \%)$ had tertiary level of education. Health workers made up 156 (12.7\%) respondents. The summary statistics of the socio-demographic profile of the study participants are shown in Table 1.

One thousand and two $(81.6 \%)$ respondents knew vaccines

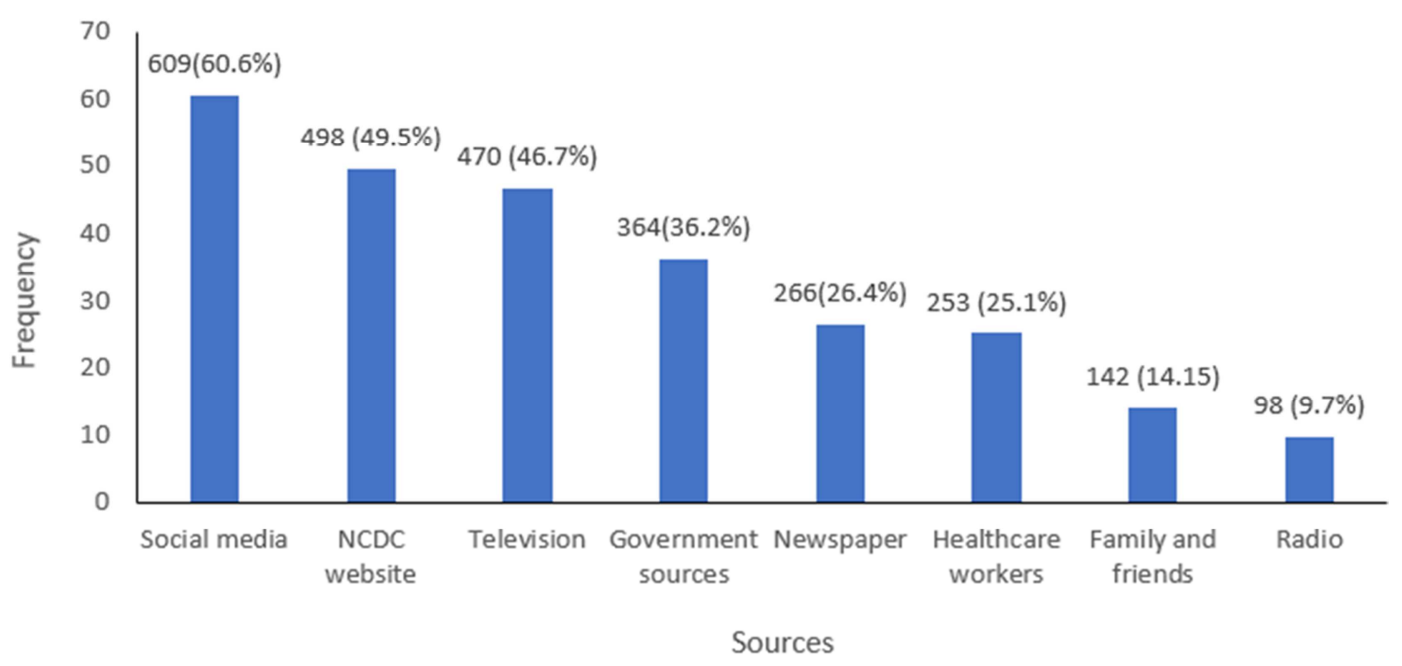

Figure 1. Source of information on COVID-19 (multiple responses).

Out of the 1,228 respondents that participated in the study, $669(54.5 \%)$ believed that a COVID-19 vaccine when available in the country should be made compulsory for school children, $775(63.1 \%)$ for health care workers, $593(48.3 \%)$ for the elderly population, $532(43.3 \%)$ for pregnant women and 583 $(47.5 \%)$ for persons with pre-morbid conditions.

Six hundred and seventeen $(50.2 \%)$ respondents were willing to accept a COVID-19 vaccine when one becomes available in the country (See reasons for participating as presented in Figure 2) while for the 611 respondents who expressed unwillingness, the reasons provided are also shown protect individuals against infectious diseases, $962(78.3 \%)$ knew vaccines strengthen the immune system, 980 (79.8\%) knew vaccination stops the spread of disease. In all, 988 $(80.5 \%)$ were assessed to have a good general knowledge of vaccination. One thousand and six $(81.9 \%)$ were aware of the development of a COVID-19 vaccine. The main source of COVID-19 vaccine-related information was social media 609 $(60.6 \%)$ followed by Nigeria Centre for Disease Control (NCDC) website, 498 (49.5\%). [Figure 1]

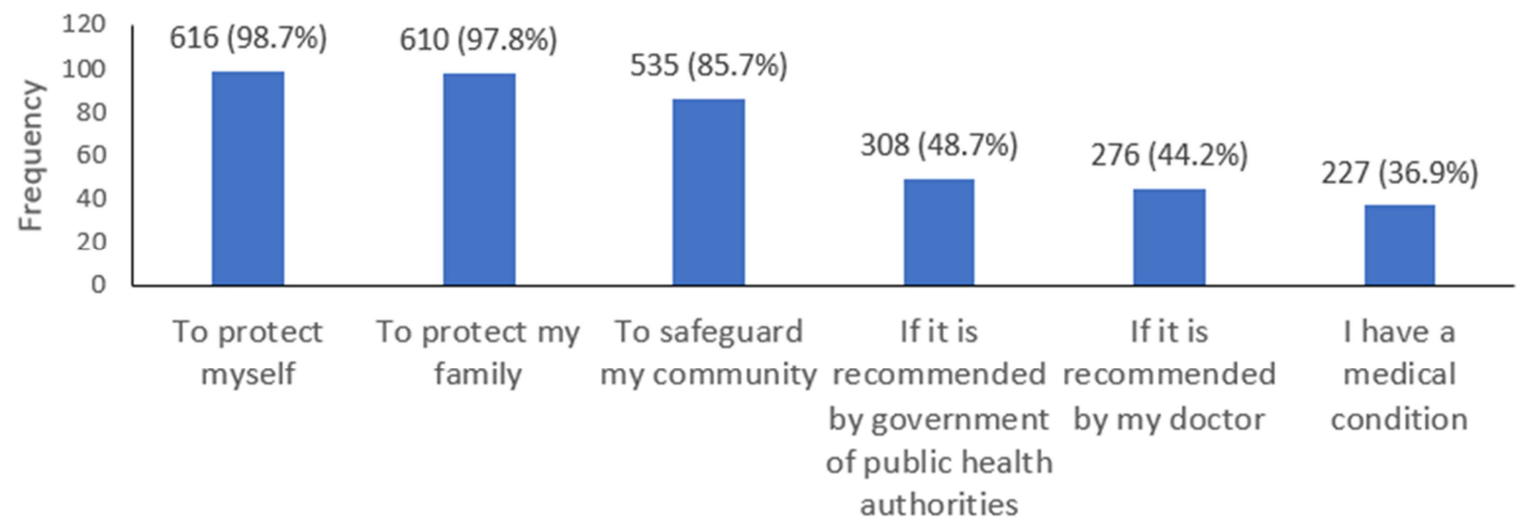

Reasons

Figure 2. Reasons for vaccine acceptance (multiple responses). 


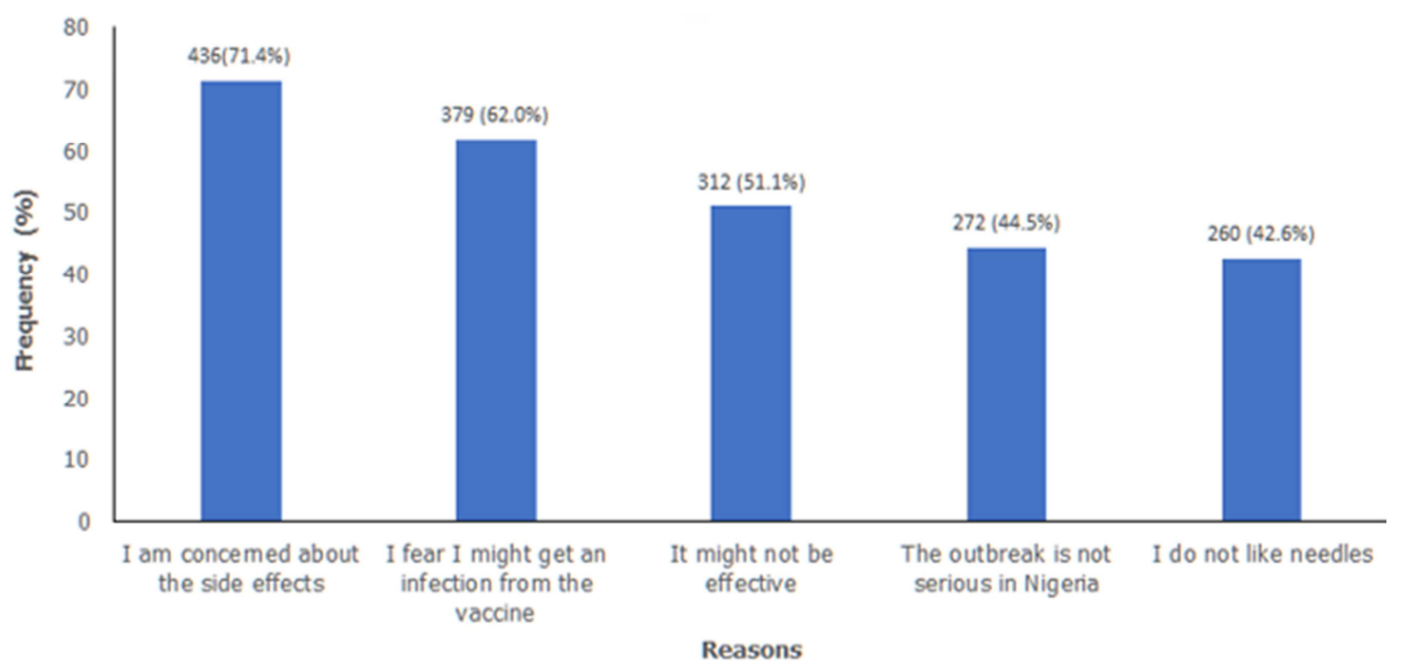

Figure 3. Reasons for vaccine rejection (multiple responses).

Perception of risk was very high for 218 (17.8\%), high for 288 (23.5\%), low for $340(27.7 \%)$ and no risk for $382(31.1 \%)$ respondents. Healthcare workers had a significantly higher perception of risk, as $56.4 \%(88 / 156)$ of them compared to $39.0 \%(418 / 1072)$ of non-healthcare workers felt they were of high/very high risk of infection $(\chi 2=17.05, \mathrm{P}<0.001)$.

Five hundred and thirty-five (43.6\%) and 451 (36.7\%) would be willing to travel for at least an hour and be willing to pay for a COVID-19 vaccine, respectively. Five hundred and eight $(41.4 \%)$ respondents expressed trust in the federal government to take the right decision regarding COVID-19 vaccination in Nigeria, $720(58.6 \%)$ held a contrary opinion. Seven hundred and twenty -nine (59.4\%) respondents felt they could trust their public health authorities regarding a COVID-19 vaccine, $761(62.0 \%)$ respondents could trust COVID-19 vaccine producers. Four hundred and ninety $(39.9 \%)$ considered the availability of a COVID-19 vaccine as a necessity for the restoration of a pre-outbreak way of life.

Respondents who were willing to travel for at least an hour to get a COVID-19 vaccine were 6.70 times more likely to accept a vaccine when it becomes available in the country compared to those who would not be willing to travel. In the same manner, those who expressed they would be willing to pay for a COVID-19 vaccine were 3.69 more likely to accept a vaccine than those who would not be willing to pay. Respondents who expressed trust in government and trust in public health authorities were 3.35 times and 2.21 times likely to accept a vaccine compared to those who held mistrust for government and public health authorities respectively $(\mathrm{P}<0.001,95 \%$ CI 2.40-4.67 and $\mathrm{P}<0.001$ 95\% CI 1.56- 3.12 respectively). Risk perception and confidence in the genuineness of vaccine producers had no significant effect on willingness to accept a vaccine.

Table 2. Multivariate analysis of sociodemographic characteristics and willingness to take a future COVID-19 vaccine ( $n=1,228)$.

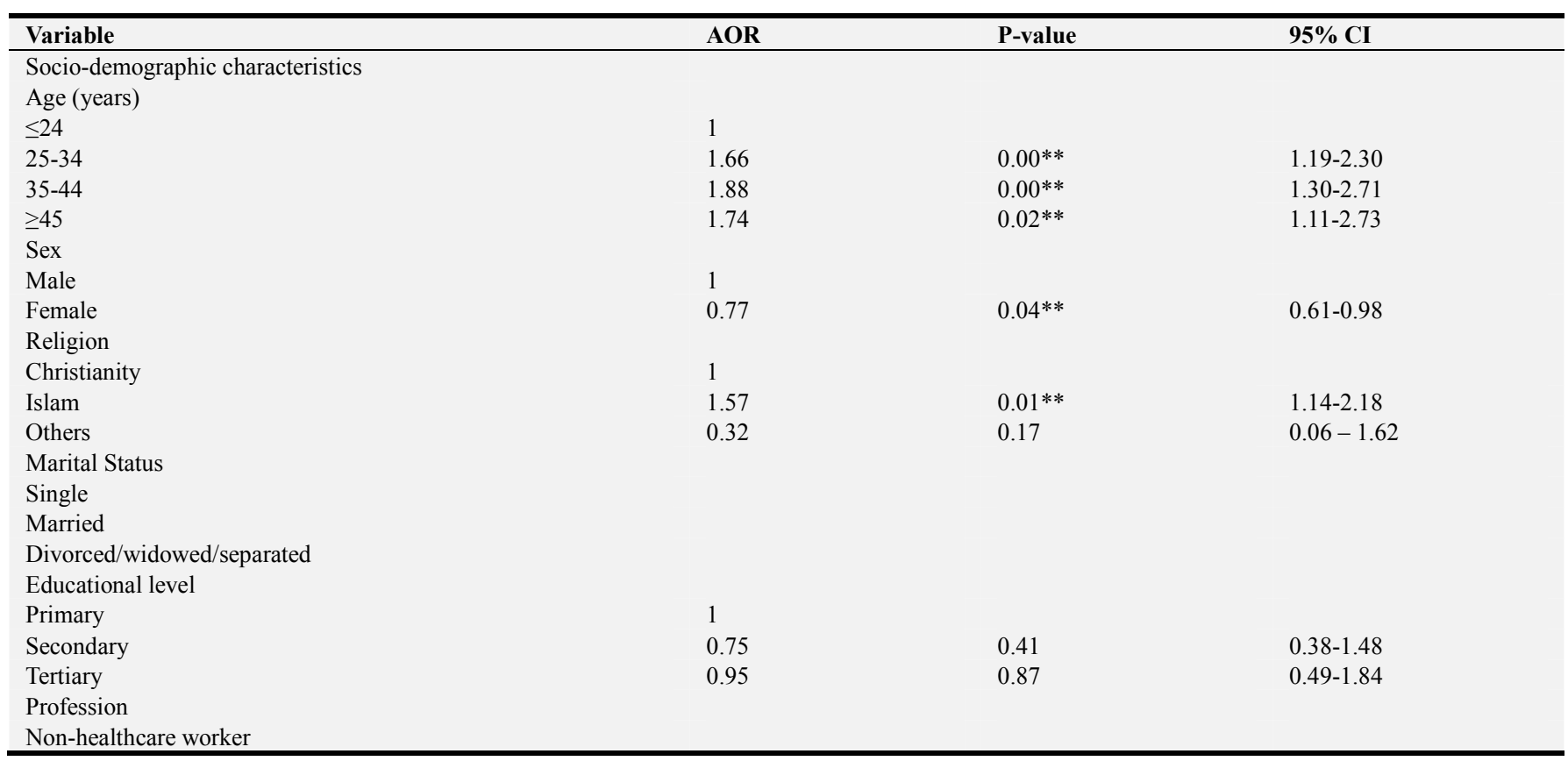




\begin{tabular}{llll}
\hline Variable & AOR & P-value & 95\% CI \\
\hline Healthcare worker & & & \\
Employment status & 1 & & $0.46-1.00$ \\
Employed with government & 0.68 & 0.05 & $0.49-0.94$ \\
Employed in private sector & 0.68 & $0.02 * *$ & $0.98-2.10$ \\
Self-employed & 1.44 & 0.06 & \\
Unemployed & & & \\
Pre-existing illness & & & \\
No & & & \\
Yes & & \\
\hline
\end{tabular}

**significant at multivariate analysis

Five hundred and eighty-three (47.5\%) had ever received a vaccine during an outbreak. One hundred and ninety $(15.5 \%)$ respondents had ever experienced a side effect from vaccination or know someone who has. 206 (16.8\%) had ever deliberately refused or delayed vaccination for themselves or family members. In bivariate analysis, having ever received a vaccine during an outbreak was significantly associated with willingness to accept a future COVID-19 vaccine $(\mathrm{P}<0.001)$ [Table 3].

Table 3. Multivariate analysis of factors associated with willingness to take a future COVID-19 vaccine $(n=1,228)$.

\begin{tabular}{|c|c|c|c|}
\hline Variable & AOR & P-value & $95 \% \mathrm{CI}$ \\
\hline \multicolumn{4}{|l|}{ Self-perception of risk } \\
\hline Low risk/No risk & 1 & & \\
\hline High risk/ Very high risk & 0.8 & 0.19 & $0.58-1.11$ \\
\hline \multicolumn{4}{|c|}{ Willingness to travel for more than one hour to get a vaccine } \\
\hline No & 1 & & \\
\hline Yes & 6.70 & $0.00 * *$ & $4.58-9.87$ \\
\hline \multicolumn{4}{|l|}{ Willingness to pay } \\
\hline Yes & 3.69 & $0.00 * *$ & $2.48-5.49$ \\
\hline \multicolumn{4}{|l|}{ Trust in government } \\
\hline No & 1 & & \\
\hline Yes & 3.35 & $0.00 * *$ & $2.40-4.67$ \\
\hline \multicolumn{4}{|c|}{ Trust in public health authorities } \\
\hline No & 1 & & \\
\hline Yes & 2.21 & $0.00 * *$ & $1.56-3.12$ \\
\hline Yes & 1.07 & 0.71 & $0.75-1.52$ \\
\hline
\end{tabular}

**significant at multivariate analysis

\section{Discussion}

The spread of the pandemic and the resultant effects on public health and the global economy in the absence of a definitive cure has heightened the demand for a vaccine and progress towards vaccine development. Vaccine hesitancy poses a real threat, as adequate coverage levels are required to stop transmission of the virus. This study examined the willingness to accept a future COVID-19 vaccine.

Most respondents were aware that COVID-19 vaccines were under development, probably because social media, the most frequent source of vaccine information for respondents, is rift with vaccine production debates including conspiracy theories. The low patronage of government websites is a reflection of the reduced trust in government with regards to COVID-19 vaccine. About a quarter of respondents sourced information from health providers, a worrisome finding, as research has shown that the public's willingness to accept a vaccine was linked to recommendations from a health provider. $[11,12]$ Health professionals should be engaged in community messaging to improve trust in a COVID-19 vaccine and increase uptake when one becomes available in the country.

Some groups have been identified as having an increased risk of infection with COVID-19 including the elderly and persons with comorbid conditions. The study showed that slightly less than half of the respondents were in favour of vaccines for the elderly and persons with health conditions, and slightly above half for school children. Health communication during planning for a vaccine roll-out in Nigeria should address these opinions, as they will impact the willingness of families to bring elderly parents and children for vaccination.

About one in two respondents were willing to take a COVID-19 vaccine, this was comparable to findings from China, $64.0 \%$ [12], Italy $86.1 \%$ [11], Australia $85.5 \%$ [13] and United States $67.0 \%$ [14]. This contrasts with Poland where willingness to accept a vaccine was $31.3 \%$ [15] Saudi Arabia 44.7\% [16] and France 47.6\%. [17] The differences may be a factor of the time during the pandemic when the studies were conducted, as studies conducted early in the outbreak when 
the virus was still poorly understood may differ from those conducted when the public had a clearer understanding of the disease.

Being an older adult was a predictor of COVID-19 vaccine acceptance, and has been documented in other studies [5, 17] and contrasts with a study in Saudi Arabia where younger people were willing to accept a vaccine [16]. While older adults have an increased risk of mortality after infection, younger persons may hold the opinion that they are healthy and do not need vaccination yet are more likely to be asymptomatic carriers and spreaders.

Males were also more likely to accept a vaccine as in other studies [5, 13], contrary to a Polish study where an affirmative response was more from females. [15] Although epidemiological information about the disease shows males are more infected than females, heath education should target females.

Interestingly, risk perception was not associated with vaccine acceptance in contrast to other studies. [8, 13, 17] This finding may be a reflection of the general poor-compliance of the public with government regulations on non-pharmacological interventions such as the compulsory use of face mask, physical and social distancing in public places, hand washing practices and the use of alcohol-based hand sanitisers.

The lack of a statistically significant difference in vaccine acceptance between healthcare workers and non-healthcare workers requires further investigation, as the higher perception of risk among healthcare workers may lead one to conclude that they would be more willing to take a vaccine. This finding has also been documented. [11] It contrasts with findings from China. [8, 18] Healthcare workers are a high-risk group for infection due to their close interactions with sick persons and should be prioritized for a COVID-19 vaccination. Further studies are required to investigate the reasons for this observation among healthcare workers, as mistrust of government and the entire politics surrounding vaccination as well as the fear of adverse events following vaccination flooding the internet might have influenced the willingness to take the vaccine among healthcare workers and the general population studied.

Christians were less likely than Moslems to accept a vaccine probably because of the widespread conspiracy theory that has been promoted by social media and reputable church leaders. Religious leaders therefore should not only be actively engaged in the planning and implementation phase of any COVID-19 vaccine intervention in the country but be encouraged to support positive messaging and role modelling.

Self-employed respondents were found to have significantly lower vaccine acceptance. This may not be unconnected to the lack of health insurance for the self-employed and therefore high -out of pocket expenditure on health, and the concern that the vaccine may not be without a cost. Further studies are required on the barriers self-employed persons may face in accepting a vaccine.

Personal and family protection were popular reasons given by respondents for accepting a vaccine. These reasons have also been elucidated in other studies. [15] The concerns about a future vaccine's effectiveness and safety raised by respondents who expressed unwillingness have been documented in other studies. [13, 17, 18] Public health authorities can act on this information by developing intentional messaging to the public. Accessibility and affordability were identified as significant predictors of willingness to accept a vaccine and should be considered when planning to introduce the vaccine into the country.

Trust in government and public health authorities were lower than was reported in other studies [13] and was a predictor of acceptance of a vaccine, with higher trust in government significantly increased likelihood of vaccine acceptance. [19] The country has been plagued in recent years with growing distrust in government, manifested in the disbelief in COVID-19 and insinuations that the disease was a government scam. [20] Building public confidence in government and health authorities will be crucial for successful vaccine uptake in the country and should include targeted messaging and community engagement.

The finding that 1 in 2 Nigerians would accept a vaccine is encouraging as herd immunity through vaccination requires a sufficient proportion of the population to be vaccinated [5]. The herd immunity threshold, calculated as $1-1 / \mathrm{R}_{0}$ (where $\mathrm{R}_{0}$ is the basic reproductive number) is the population proportion that should be immune to prevent transmission of infectious disease, and for COVID-19 is estimated to be between 55\% and $82 \%$ [14]. In Nigeria, $R_{0}$ is 2.42 [21] leaving herd immunity threshold as $59 \%$.

The study has several limitations that may restrict the generalizability of the study findings. Being an online survey, selection bias could have been introduced in the sampling technique as respondents who had no access to the internet may be different from the general population. Acceptance was assessed using a hypothetical vaccine and findings may differ from what would have been obtained if a vaccine was existent in the country. It may therefore be useful to repeat the survey when there is a vaccine in-country.

\section{Conclusion}

One in two respondents studied expressed their willingness to accept a COVID-19 with age $>24$ years, Islamic religion, male gender, trust in government, trust in public health authorities, confidence in vaccine developers, willingness to pay for and travel for a vaccine, and vaccination during an outbreak identified as significant determinants for COVID-19 vaccine acceptance. Potential side effects from COVID-19 vaccine was a major concern relating to vaccine hesitancy. Strategies to introduce a COVID-19 vaccine into the country should build on the finding from this study to target groups at high risk of hesitancy, improve public trust in government and health authorities, and the rollout of sensitization activities involving all relevant stakeholders on vaccine effectiveness, accessibility and cost. 


\section{Acknowledgements}

The authors are grateful to all the respondents. The authors also appreciate Amen Anabue for proof-reading the manuscript.

\section{References}

[1] Unal, M. and Irez, T. (2020) COVID 19 Disease Caused by Coronavirus 2 (SARS-CoV-2) (Severe Acute Respiratory Syndrome). Asian Journal of Medicine and Health, 1-11.

[2] Mahar, I. (2020) Impact of Covid-19 on Global Economy Structure [Internet]. Modern Diplomacy. 2020 [cited 2020 Jun 14]. Available from: https://moderndiplomacy.eu/2020/04/22/impact-of-covid-19-o n-global-economy-structure/.

[3] Olum, R., Chekwech, G., Wekha, G., Nassozi, D. R. and Bongomin, F. (2020) Coronavirus Disease-2019: Knowledge, Attitude, and Practices of Health Care Workers at Makerere University Teaching Hospitals, Uganda. Frontiers in Public Health, Frontiers Media SA. 8. https://doi.org/10.3389/fpubh.2020.00181

[4] Organisation, W. H. Coronavirus [Internet].

[5] Neumann-Böhme, S., Nirosha, ·, Varghese, E., Sabat, · Iryna, Pedro, ', Barros, P. et al. (2020) Once we have it, will we use it? A European survey on willingness to be vaccinated against COVID-19. The European Journal of Health Economics, 1, 3. https://doi.org/10.1007/s10198-020-01208-6.

[6] National Bureau of Statitstics. (2018) Nigeria national immunization coverage survey (nics): national brief.

[7] Jegede, A. S. (2007) What led to the Nigerian boycott of the polio vaccination campaign? PLoS Medicine, Public Library of Science. 4 , https://doi.org/10.1371/journal.pmed.0040073.

[8] Harapan, H., Wagner, A. L., Yufika, A., Winardi, W., Anwar, S. Gan, A. K. et al. (2020) Acceptance of a COVID-19 Vaccine in Southeast Asia: A Cross-Sectional Study in Indonesia. Frontiers in Public Health, Frontiers. 8, 381. https://doi.org/10.3389/fpubh.2020.00381.

[9] Domek, G. J., O’Leary, S. T., Bull, S., Bronsert, M., Contreras-Roldan, I. L., Bolaños Ventura, G. A. et al. (2018) Measuring vaccine hesitancy: Field testing the WHO SAGE Working Group on Vaccine Hesitancy survey tool in Guatemala Vaccine, Elsevier Ltd. 36, 5273-81. https://doi.org/10.1016/j.vaccine.2018.07.046.

[10] Ma, X., He, Z., Wang, Y., Jiang, L., Xu, Y., Qian, C. et al. (2011) Knowledge and attitudes of healthcare workers in Chinese intensive care units regarding $2009 \mathrm{H} 1 \mathrm{~N} 1$ influenza pandemic. BMC Infectious Diseases, BioMed Central. 11, 1-7. https://doi.org/10.1186/1471-2334-11-24.
[11] Barello, S., Nania, T., Dellafiore, F., Graffigna, G. and Caruso, R. (2020) 'Vaccine hesitancy' among university students in Italy during the COVID-19 pandemic. European Journal of Epidemiology, Springer. 1,1 . https://doi.org/10.1007/s10654-020-00670-z.

[12] Sun, S. (2020) Interest in COVID-19 vaccine trials participation among young adults in China: Willingness, reasons for hesitancy, and demographic and psychosocial determinants. Med Rxiv: the preprint server for health sciences, 2020.07.13.20152678.

https://doi.org/10.1101/2020.07.13.20152678.

[13] Dodd, R. H., Cvejic, E., Bonner, C., Pickles, K. and Mccaffery, K. J. (2014) Willingness to vaccinate against COVID-19 in Australia. BMC Public Health, 14, 484 https://doi.org/10.1016/S1473-3099(20)30559-4.

[14] Malik, A. A., McFadden, S. M., Elharake, J. and Omer, S. B. (2020) Determinants of COVID-19 vaccine acceptance in the US. EClinical Medicine, Elsevier. 0, 100495. https://doi.org/10.1016/j.eclinm.2020.100495.

[15] Malesza, M. (2020) Acceptability of COVID-19 vaccination during pandemic phase of coronavirus in Poland: population-based cross-sectional survey.

[16] Magadmi, R. and Kamel, F. (2020) Beliefs and Barriers Associated with COVID-19 Vaccination Among the General Population in Saudi Arabia. Research Square.

[17] Detoc, M., Bruel, S., Frappe, P., Botelho-Nevers, E. and Gagneux-Brunon, A. (2020) Intention to participate in a COVID-19 vaccine clinical trial and to get vaccinated against COVID-19 in France during the pandemic. MedRxiv, 2020.04.23.20076513. https://doi.org/10.1101/2020.04.23.20076513.

[18] Fu, C., Wei, Z., Pei, S., Li, S., Sun, X. and Liu, P. (2020) Acceptance and preference for COVID-19 vaccination in health-care workers (HCWs). https://doi.org/10.1101/2020.04.09.20060103.

[19] Padhi, B. K. and A. Almohaithef, M. (2020) Determinants of intent to uptake Coronavirus vaccination among respondents in Saudi Arabia: a web-based national survey. MedRxiv, 2020.05.27.20114413. https://doi.org/10.1101/2020.05.27.20114413.

[20] Omaka-Amari, L., Aleke, A., Obande-Ogbuinya, N., Ngwakwe, P., Nwankwo, O. and Afoke, E. (2020) Coronavirus (COVID-19) Pandemic in Nigeria: Preventive and Control Challenges within the First Two Months of Outbreak. African Journal of Reproductive Health, 24, 87-97.

[21] Adekunle, A. I., Adegboye, O. A., Gayawan, E. and McBryde, E. S. (2020) Is Nigeria really on top of COVID-19? Message from effective reproduction number. Epidemiology and Infection, NLM (Medline). 148, e166. https://doi.org/10.1017/S0950268820001740. 